\title{
Accuracy of Measuring Camera Position by Marker Observation
}

\author{
Vladimir A. Grishin \\ Space Research Institute (IKI), Russian Academy of Sciences, Moscow, Russia. \\ Email: vgrishin@iki.rssi.ru \\ Received July $14^{\text {th }}, 2010$; revised August $10^{\text {th }}, 2010$; accepted August $14^{\text {th }}, 2010$.
}

\begin{abstract}
A lower bound to errors of measuring object position is constructed as a function of parameters of a monocular computer vision system (CVS) as well as of observation conditions and a shape of an observed marker. This bound justifies the specification of the CVS parameters and allows us to formulate constraints for an object trajectory based on required measurement accuracy. For making the measurement, the boundaries of marker image are used.
\end{abstract}

Keywords: Computer Vision System, Camera Position Measurement, Marker Observation, Lower Bound to Errors

\section{Introduction}

CVSs are widely applied for a solution of motion control problems. This fact is associated by the following conditions. First, the computational capability of available processors allows for the real-time processing of large volumes of information formed by TV cameras. The information processing time proves to be acceptable to a number of practical problems [1-6]. Second, the increasing application of computer-aided control systems of unmanned aerial vehicles requires the enhancement of the vector of measured parameters to solve the automatic landing problem [5]. Another task is a docking problem (including the spacecraft docking), which requires precise measuring a relative position for solving the terminal control task [6]. As an example we can refer to the docking the first European Automated Transfer Vehicle (ATV) "Jules Verne" to the International Space Station (ISS) on 3 April 2008. In the above experiment, a special computer vision system was used for measuring the relative spatial and angular position.

All of these facts stimulate interest in estimation of the potential accuracy (lower bounds to errors) of measuring the position parameters as a function of the marker shape, its observation condition and technical parameters of the CVS. This allows us to evaluate an applicability of CVSs to solving control problems under specific conditions as well as to optimize the CVS parameters from the viewpoint of ensuring the required accuracy of measurements. There are a small number of publications devoted to the problems of determining the current coordinates measurement precision estimation. Most publications are based on experimental approach (full-scale experiments or stochastic simulation) to the measurement precision estimation. For obtaining reliable estimation, such approach requires too much time and additionally the full-scale experiments are very expensive.

In [7], the Cramér-Rao bound is constructed to camera position estimation by docking marker observation. For position estimation, a set of the marker features (points of interest) are used, namely corners, contrast spots and others. This approach is suitable for the case of small or medium marker observation distance. In such distances the visible size of marker is of order tens or hundreds of pixels in any direction. In the present paper, we consider the approach, which is suitable for large distances by using the boundaries between marker image and background. This approach allows obtaining lower bound to errors of measuring object position with small computational expenses. It allows in one's turn to optimize CVS parameters and marker shape for a specified set of the observation conditions.

In Section 2, we formulate the assumptions for constructing the bound to errors. In Section 3, we construct a Cramér-Rao bound to the measurement errors and, in Section 4, we present experimental results.

\section{Assumptions Made When Constructing a Bound}

We make the following simplifying assumptions to esti- 
mate the methodic errors:

- The resolution of the optical system is the same over the frame area.

- There are no geometrical distortions of the optical system (or they are compensated for during the preprocessing of images).

- The optical system is calibrated during its manufacturing and the calibration error is negligible.

- The exposure time tends to zero, so smearing of the picture due to the motion of the object during shooting can be neglected.

- The precision of marker localization is limited by signal to noise ratio.

- The parameters of this noise law are the same over the area of a frame.

- The pixel size of CCD matrix tends to zero.

All of these assumptions, except for the last one, are quite easily realizable at moderate cost. In regard to the last assumption, it is introduced for simplification of analysis. Without this simplification, an analytical solution is very difficult. Apparently, it is possible to obtain some asymptotic estimations of additional object position measurement errors, which is conditioned by limited size of CCD matrix pixels. In any case, this problem should be a subject of separate analysis. Thus, the used model has no error sources except for the image noise.

\section{Cramér-Rao Bound to Measuring Errors}

The construction and application of a likelihood function and Cramér-Rao bound for measurement errors are extensively described in the literature [8-10] and others. A likelihood function is used for constructing the Cramér-Rao lower bound to the variance of estimated parameters. The schematic view of the marker shooting is shown in Figure 1. The marker is placed in the coordinate's origin.

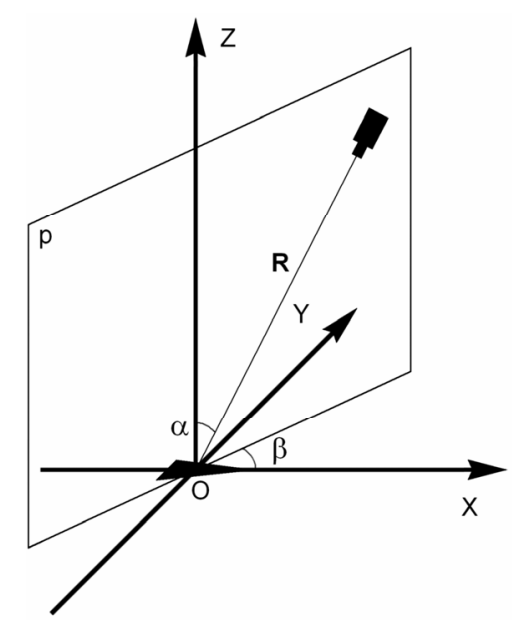

Figure 1. TV camera position.
The optical system forms the image of observed marker in the plane of a CCD matrix. The space position of the TV camera and its orientation gives a vector of parameters A that should be estimated. Camera coordinate system is shown in the Figure 2. Projection center C of the camera is placed on the end of vector $\mathbf{R}$ (Figure 1), which is turned with respect to a normal of the surface of marker on angle $\alpha$ in the plane $p$ which pass through axis $\mathrm{OZ}$ and is preliminarily rotated on the azimuth on angle $\beta$ relatively plane XOZ.

In the initial camera position vectors $\mathbf{e}_{1}, \mathbf{e}_{2}$ and $\mathbf{e}_{3}$ are given by the coordinates as follows:

$$
\begin{aligned}
& \mathbf{e}_{1}=(0,0,-1) \\
& \mathbf{e}_{2}=(-1,0,0) \\
& \mathbf{e}_{3}=(0,1,0) .
\end{aligned}
$$

The above three vectors are rotated by an angle $\alpha$ together with the projection center of camera $\mathrm{C}$ in the plane $p$. So, the obtained coordinates of the vectors are the following:

$$
\begin{aligned}
& \mathbf{e}_{1}=\left(e_{11}, e_{12}, e_{13}\right) \\
& \mathbf{e}_{2}=\left(e_{21}, e_{22}, e_{23}\right) \\
& \mathbf{e}_{3}=\left(e_{31}, e_{32}, e_{33}\right) .
\end{aligned}
$$

Let $\gamma, \varphi$ and $\psi$ be three rotation angles around the vectors $\mathbf{e}_{1}, \mathbf{e}_{2}$ and $\mathbf{e}_{3}$ respectively. The first rotation is the rotation by the angle $\gamma$. Since the TV camera is space stabilized so that, the image of observed marker is in the center of the vision area, it is possible to suppose the angles $\varphi$ and $\psi$ small enough $(\varphi \approx 0, \psi \approx 0)$. Hence, the rotation operators by the angles $\varphi$ and $\psi$ are approximately commutative.

The coordinates of any $i$-th marker point $\left(X_{i}, Y_{i}, Z_{i}\right)$ taken in camera coordinate system are the following:

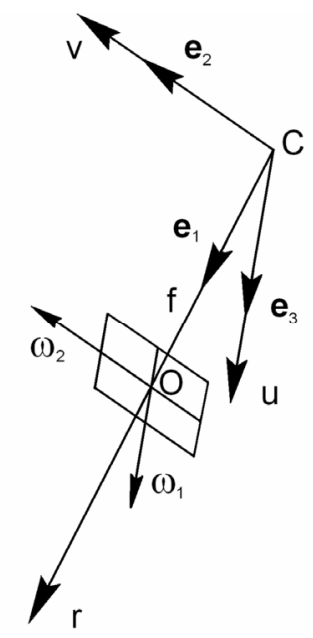

Figure 2. Camera coordinate system. 


$$
\begin{aligned}
& X_{i}^{\prime}=X_{i}-X \\
& Y_{i}^{\prime}=Y_{i}-Y \\
& Z_{i}^{\prime}=Z_{i}-Z,
\end{aligned}
$$

where $(X, Y, Z)$ is the coordinates of camera projection center $\mathrm{C}$. The coordinates of the $i$-th point of the marker in CCD matrix are calculated by:

$$
\begin{aligned}
& a=\frac{f}{X_{i}^{\prime} e_{11}+Y_{i}^{\prime} e_{12}+Z_{i}^{\prime} e_{13}} \\
& \omega_{1}^{i}=a\left(X_{i}^{\prime} e_{31}+Y_{i}^{\prime} e_{32}+Z_{i}^{\prime} e_{33}\right) \\
& \omega_{2}^{i}=a\left(X_{i}^{\prime} e_{21}+Y_{i}^{\prime} e_{22}+Z_{i}^{\prime} e_{23}\right),
\end{aligned}
$$

where $f$-is a focal distance of the camera.

For the specified camera's spatial and angular positions, the $i$-th point $\left(X_{i}, Y_{i}, Z_{i}\right)$ taken in the coordinates of CCD matrix depends on the parameters:

$$
\begin{aligned}
\omega_{1}^{i} & =\omega_{1}^{i}(X, Y, Z, \gamma, \varphi, \psi) \\
\omega_{2}^{i} & =\omega_{2}^{i}(X, Y, Z, \gamma, \varphi, \psi) .
\end{aligned}
$$

Since we consider an observation of marker from medium and long distances, the measurement angular errors of $\varphi$ and $\psi$ as well as the translation errors in the direction of the vectors $\mathbf{e}_{3}$ and $\mathbf{e}_{2}$ are heavily correlated. So, we estimate the precision only for four parameters, that are given by a vector $\tilde{\mathbf{A}}=(r, v, u, \gamma)$. Axis $r$ is parallel to $\mathbf{e}_{1}, v$ is parallel to $\mathbf{e}_{2}$ and $u$ is parallel to $\mathbf{e}_{3}$.

The construction and application of the likelihood function are well known from [8-10] and others. This likelihood function is used for constructing the Cramér-Rao lower bound to the variance of estimated parameters. The likelihood function depends on parameters being under estimation. The estimations of the parameters are defined by the values that provide the extremum of the likelihood function:

$$
P(\tilde{\mathbf{A}}) \rightarrow \text { extr },
$$

where $P(\tilde{\mathbf{A}})$ is the likelihood function.

The necessary condition of extremum is given by:

$$
\frac{\partial P(\tilde{\mathbf{A}})}{\partial A_{i}}=0, \quad i=1, \ldots, 4 .
$$

Accordingly, we can use a logarithm of the likelihood function for finding of extremum of $P(\tilde{\mathbf{A}})$. Analogous condition of extremum can be:

$$
\frac{\partial \ln P(\tilde{\mathbf{A}})}{\partial A_{i}}=0, \quad i=1, \ldots, 4 .
$$

Covariance matrix of estimated parameters is:

$$
R=J^{-1},
$$

where $J$ is the Fisher information matrix, which is calculated from the likelihood function. According to the Cramér-Rao inequalities, the lower bounds to the variances of unbiased estimation errors are given by:

$$
\begin{aligned}
& \sigma_{r}^{2} \geq R_{11}(\tilde{\mathbf{A}}), \quad \sigma_{v}^{2} \geq R_{22}(\tilde{\mathbf{A}}), \\
& \sigma_{u}^{2} \geq R_{33}(\tilde{\mathbf{A}}), \quad \sigma_{\gamma}^{2} \geq R_{44}(\tilde{\mathbf{A}}) .
\end{aligned}
$$

We estimate the covariance for the estimation of vector A. For this goal, we first determine the Fisher information matrix, which is expressed via the second derivatives of the likelihood function as follows:

$$
J_{i j}=-E\left[\frac{\partial^{2} \ln P(\tilde{\mathbf{A}})}{\partial A_{i} \partial A_{j}}\right]=E\left[\frac{\partial \ln P(\tilde{\mathbf{A}})}{\partial A_{i}} \frac{\partial \ln P(\tilde{\mathbf{A}})}{\partial A_{j}}\right],
$$

where $E[\ldots]$ is a mathematical expectation.

Let's consider an observed image of marker:

$$
\mu(\boldsymbol{\omega})=s(\boldsymbol{\omega}, \tilde{\mathbf{A}})+\xi(\boldsymbol{\omega}),
$$

where $s(\boldsymbol{\omega}, \tilde{\mathbf{A}})$ is the marker image and $\xi(\boldsymbol{\omega})$ is noise with intensity $\sigma^{2}=N_{0} / 2$. Without loss of commonness, we can suppose that a brightness value of marker image $s(\boldsymbol{\omega}, \tilde{\mathbf{A}})$ is equal to one, and a brightness of remaining part of the cadre is zero.

In reference [11], an expression of Fisher Information Matrix was derived for the case of one-dimensional signal. For the two-dimensional case, this expression can be easily obtained by the same way:

$$
J_{i j}=\frac{2}{N_{0}} E\left[\int_{\Omega} \frac{\partial s(\boldsymbol{\omega}, \tilde{\mathbf{A}})}{\partial A_{i}} \frac{\partial s(\boldsymbol{\omega}, \tilde{\mathbf{A}})}{\partial A_{j}} d \boldsymbol{\omega}\right],
$$

where $\Omega$ is a marker image area and $d \omega$ is an elementary square in $\Omega$.

In general case, the calculation of the Fisher information matrix requires to determine the above mathematical expectation $E[\ldots]$. In our case, the expression in square brackets is deterministic, and therefore we obtain the following elements of the Fisher information matrix:

$$
J_{i j}=\frac{2}{N_{0}} \int_{\Omega} \frac{\partial s(\boldsymbol{\omega}, \tilde{\mathbf{A}})}{\partial A_{i}} \frac{\partial s(\boldsymbol{\omega}, \tilde{\mathbf{A}})}{\partial A_{j}} d \boldsymbol{\omega}
$$

Let's consider derivatives. The $\tilde{\mathbf{A}}$ is the vector of parameters that gives the camera position. The finite difference approximation of the derivative is defined as follows: 


$$
\begin{aligned}
& \frac{\partial s(\boldsymbol{\omega}, \tilde{\mathbf{A}})}{\partial A_{i}} \approx \frac{\Delta s(\boldsymbol{\omega}, \tilde{\mathbf{A}})}{\Delta A_{i}}=\frac{s\left(\boldsymbol{\omega}, \tilde{\mathbf{A}}+\Delta \tilde{\mathbf{A}}_{i}\right)-s(\boldsymbol{\omega}, \tilde{\mathbf{A}})}{\Delta A_{i}}, \\
& \Delta \tilde{\mathbf{A}}_{i}=\left(0, \ldots \Delta A_{i}, \ldots .0\right) .
\end{aligned}
$$

Figure 3 shows the marker image in the initial position $s(\boldsymbol{\omega}, \tilde{\mathbf{A}})$. In Figure 4, the marker images are shown for both the shifted position $s\left(\boldsymbol{\omega}, \tilde{\mathbf{A}}+\Delta \tilde{\mathbf{A}}_{i}\right)$ and the initial position $s(\boldsymbol{\omega}, \tilde{\mathbf{A}})$. The gray colours of different intensity are used for marking difference between both these images.

The difference can be calculated by integrating an optical flow on the contour of marker as follows:

$$
\frac{\partial s(\boldsymbol{\omega}, \tilde{\mathbf{A}})}{\partial A_{i}}= \begin{cases}\left(\mathbf{n}, \mathbf{Q}_{i}\right), & \boldsymbol{\omega} \in C \\ 0, & \boldsymbol{\omega} \notin C,\end{cases}
$$

where $\mathbf{n}$ is the external normal $(\|\mathbf{n}\|=1)$ with respect to the marker image boundary (contour), $\mathbf{Q}_{i}$ is the optical flow, which is caused by $\Delta \tilde{\mathbf{A}}_{i},\left(\mathbf{n}, \mathbf{Q}_{i}\right)$ is a scalar product of the vectors $\mathbf{n}$ and $\mathbf{Q}_{i}$ and $\mathrm{C}$ is the marker boundary. In such a way, we show that the surface integral (1) is reduced to the following contour integral:

$$
J_{i j}=\frac{2}{N_{0}} \int_{C}\left(\mathbf{n}, \mathbf{Q}_{i}\right)\left(\mathbf{n}, \mathbf{Q}_{j}\right) d l
$$

Thus we have obtained the expression for any element of the Fisher information matrix. For the one segment, the integral (2) can be numerically calculated, for instance, by the trapezium method:

$$
p_{i j}=\frac{2}{N_{0}} \frac{1}{2} \sum_{n=1}^{N-1}\left(\left(\mathbf{n}, \mathbf{Q}_{i}^{n}\right)\left(\mathbf{n}, \mathbf{Q}_{j}^{n}\right)+\left(\mathbf{n}, \mathbf{Q}_{i}^{n+1}\right)\left(\mathbf{n}, \mathbf{Q}_{j}^{n+1}\right)\right) \delta l
$$

Figure 5 explains the calculation of scalar product $\left(\mathbf{n}, \mathbf{Q}_{i}\right)$. The calculation of $\left(\mathbf{n}, \mathbf{Q}_{j}\right)$ is made similarly. The $\delta q$ is the difference between scalar product $\left(\mathbf{n}, \mathbf{Q}_{i}\right)$ in the integral (2) for this segment, and in the expression (3) for this segment. Notice that $\delta q$ is proportional to $\left(\Delta A_{i}\right)^{2}$ and tends to zero in condition of $\Delta A_{i} \rightarrow 0$. So we can neglect this term. Calculation of the expression (3) should be performed for all sections of the maker boundary.

\section{Experimental Results}

To illustrate the application of the obtained relations, we estimated the errors of calculating position parameters for the marker shown in Figure 6. The marker is given by the isosceles triangle. The base of the triangle equals to two meters and its height equals to three meters. The triangle has the round spot in his centre. Contour (boundary) $C$ of this marker includes both external boundary of this triangle and internal boundary of the spot in the triangle centre.

Let's specify the following camera parameters. The focal distance of the optical system is $18 \mathrm{~mm}$. The field of camera view is $23.23^{\circ} \times 23.23^{\circ}$. Errors of position are calculated for a set of values of angle $\alpha$ : $\alpha=5,15,25,35,45,55,65^{\circ}$ (7 values) and set of values of angle $\beta$ : $\beta=0,10,20, \ldots . .350^{\circ}$ (36 values). The distance of the marker observation is $r=50 \mathrm{M}$. We put a noise intensity to be equal to $0.2(\sigma=0.2)$.

Figure 7 shows the calculation results for the mean square errors of coordinates and normalized correlation. The coordinates are measured in meters and the values of angles are measured in degrees. The errors are given by the appropriate surfaces over the matrix of size $7 \times 36$ samples, where the matrix sizes are determined by the sets of $\alpha$ and $\beta$ values respectively.

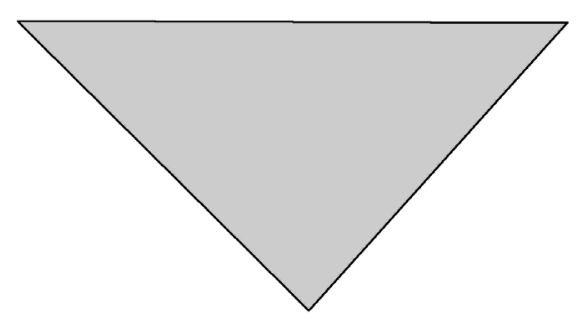

Figure 3. Triangle marker.

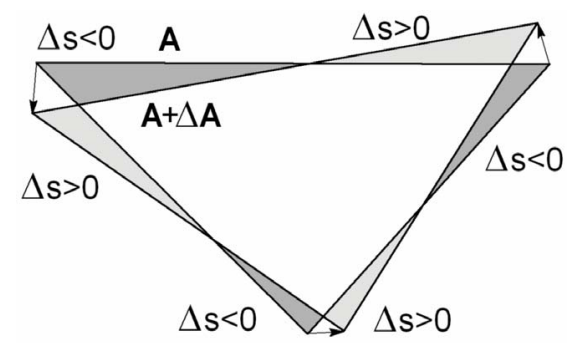

Figure 4. Difference $s\left(\boldsymbol{\omega}, \tilde{\mathbf{A}}+\Delta \tilde{\mathbf{A}}_{i}\right)-s(\boldsymbol{\omega}, \tilde{\mathbf{A}})$.

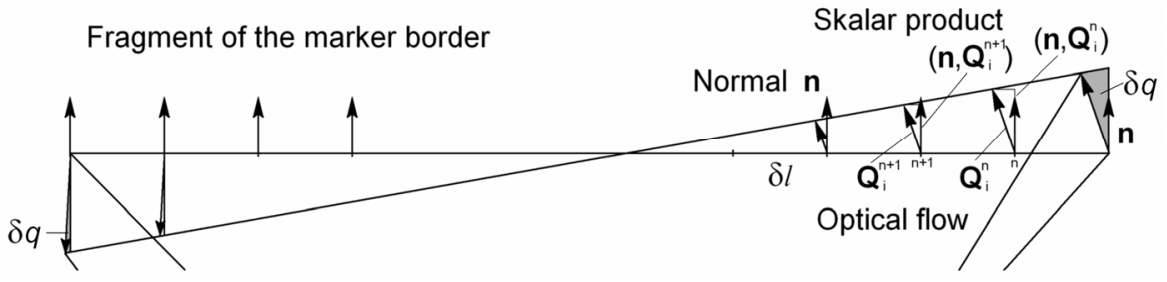

Figure 5. Calculation of the scalar product on the one segment of marker boundary. 


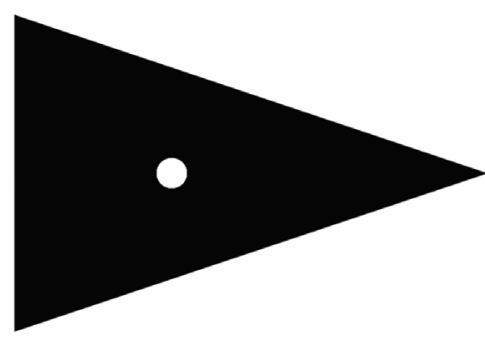

Figure 6. Marker shape.

Accordingly to Figure 7, for the distance of $50 \mathrm{~m}$ and the noise intensity $\sigma=0.2$, the range $|\mathbf{r}|$ can be measured with error $\sigma_{r}=0.02-0.04 \mathrm{~m}$, as well as the displacement in a CCD matrix plane can be measured with errors $\sigma_{V}, \sigma_{U}=0.05-0.4 \mathrm{~m}$. Rotation around the vector $\mathbf{r}$ can be measured with the error $\sigma_{\gamma}=0.015-0.04^{\circ}$. As followed from Figure 7, the functional dependences of measurement errors and normalized correlation of linear and angular coordinates are very complicated functions. We have considered the maker of uniform
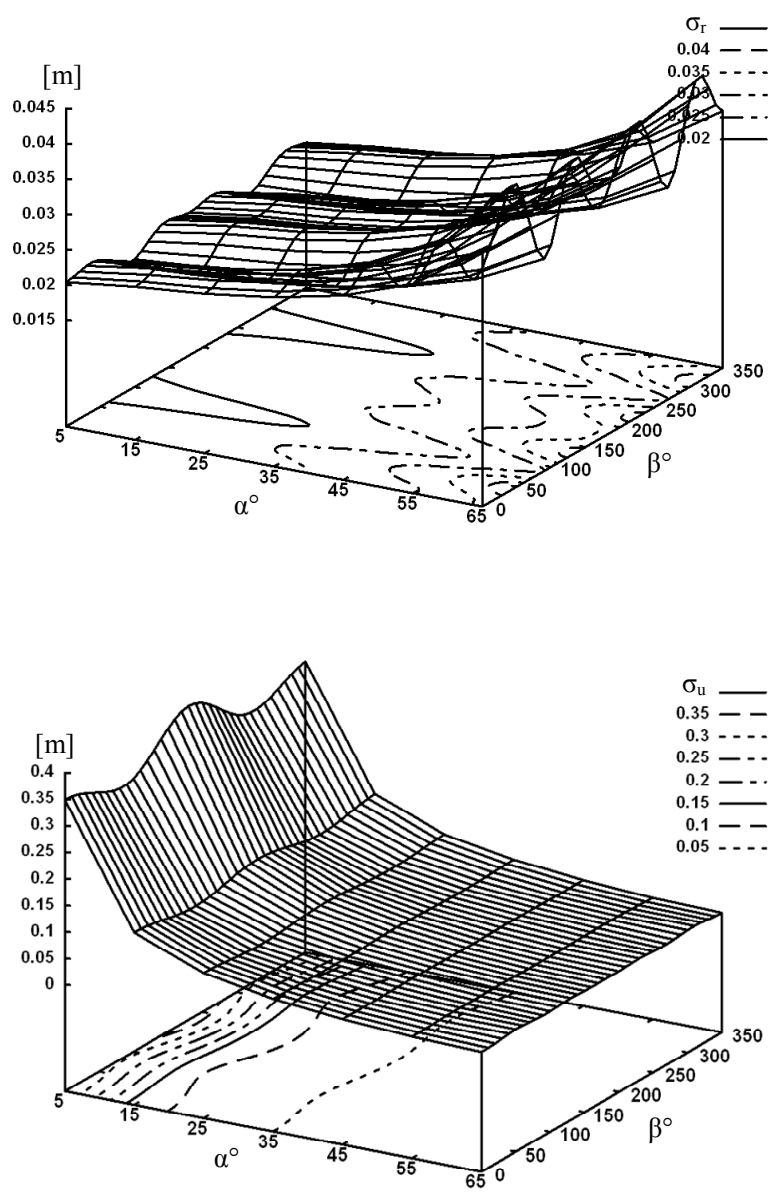

brightness. In this case, only the contrast boundary operates in the marker image. The calculated precision values are much higher than the similar values in [7] that are based on using a small set of features (points of interest) of the marker. Using the boundaries of marker image for measurement provides an increase of the measurement precision. Mention should be made that optical system distortions and low resolution of CCD camera can seriously deteriorate the precision of measurement. Joint analysis of noise and camera resolution influence on the precision of measurement is complicated enough.

The above values of the mean square error and the normalized correlation should be taken in an account in creating the computer vision system. The significant values of the normalized correlation show the considerable dependences between control loops of object position coordinates. This fact should be taken into account in the control system. The development of a computer vision system should be carried out together with the development of the marker shape.
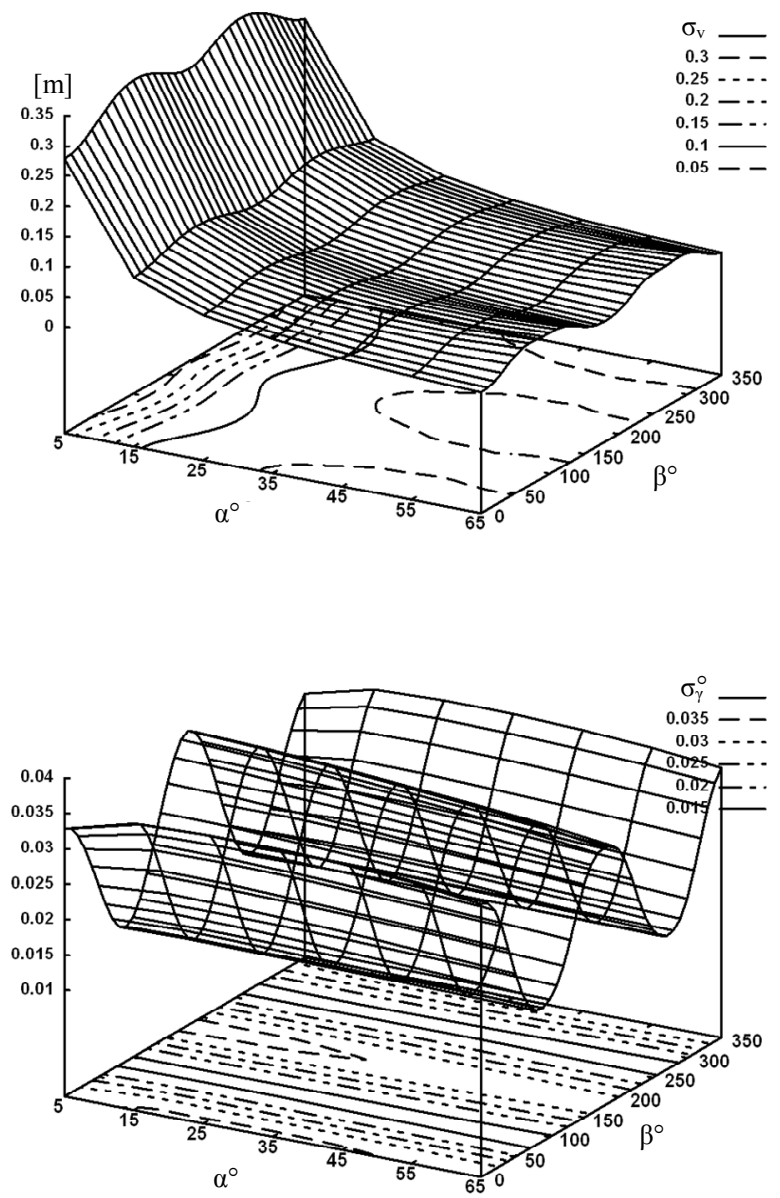

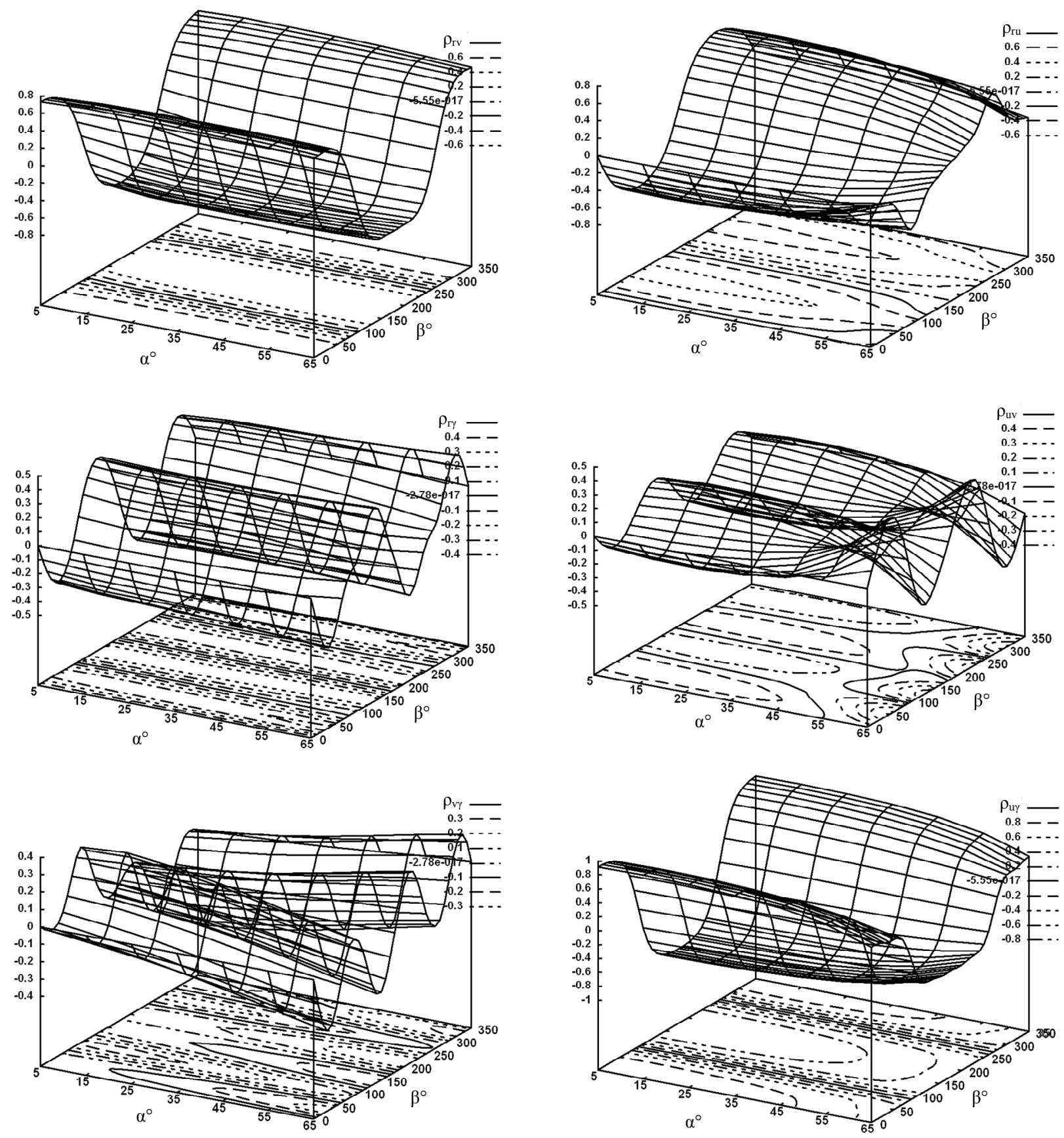

Figure 7. Errors of estimated parameters and correlation bonds between them (normalized correlation).

For comparison, we estimated the errors of calculating position parameters for the T-shaped marker shown on Figure 8.

This marker has the same area as the marker on Figure 6. Figure 9 shows the calculation results for the measurement error of coordinates.

Accordingly to Figure 9, the T-shaped marker provides a slightly higher precision of position parameters' measurement.

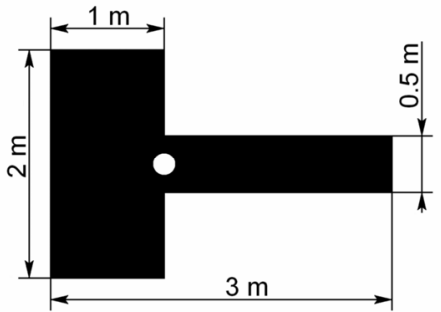

Figure 8. T-shaped marker. 

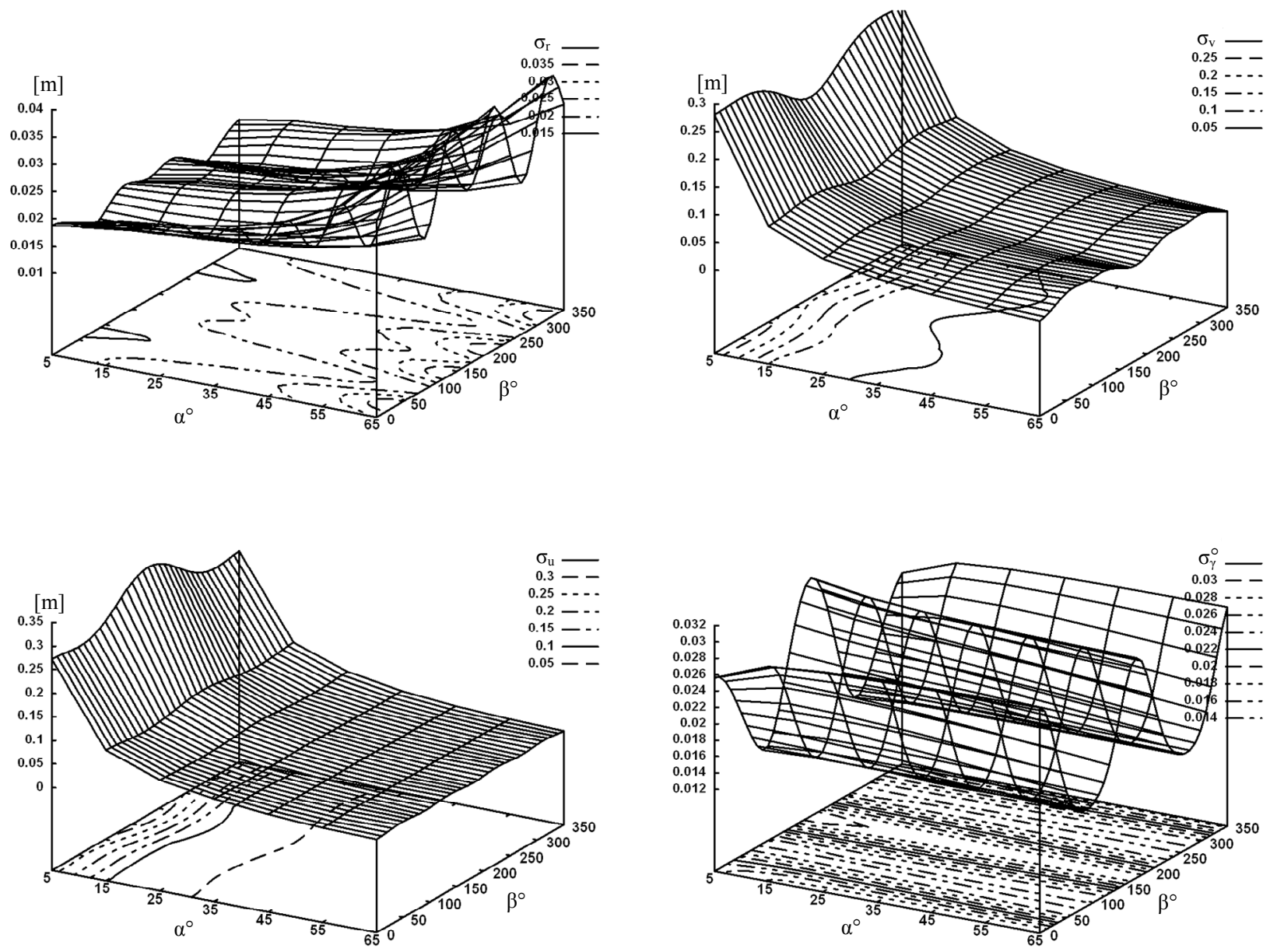

Figure 9. Errors of estimated parameters for T-shaped marker.

\section{Conclusions}

The new method has been proposed for estimating the errors of determining the TV camera position. This method is based on using the marker image of a given shape. The method allows us to estimate the measurement errors depending on shooting conditions and CVS parameters. The obtained error's estimations are useful for development of CVSs and particularly for optimization of their parameters.

\section{Acknowledgement}

This work was supported by the Russian Foundation for Basic Research, project no. 09-01-00573-a.

\section{REFERENCES}

[1] C. De Wagter and J. A. Mulder, "Towards Vision-Based UAV Situation Awareness," Proceedings of the AIAA Guidance, Navigation, and Control Conference, San
Francisco, California, August 2005, pp. AIAA-20055872.

[2] S. Saripalli, J. F. Montgomery and G. S. Sukhatme, "Visually-Guided Landing of an Unmanned Aerial Vehicle," IEEE Transactions on Robotics and Automation, Vol. 19, No. 3, June 2003, pp. 371-380.

[3] S. Saripalli and G. S. Sukhatme, "Landing a Helicopter on a Moving Target," Proceedings of IEEE International Conference on Robotics and Automation (ICRA'2007), Roma, Italy, April 2007, pp. 2030-2035.

[4] T. Kubota, S. Sawai, T. Misu, T. Hashimoto, J. Kawaguchi and A. Fujiwara, "Autonomous Landing System for MUSES-C Sample Return Mission," Proceedings of the Fifth International Symposium on Artificial Intelligence, Robotics and Automation in Space (ISAIRAS'99), ESA SP-440, Noordwijk, The Netherlands, June 1999, pp. 615-620.

[5] C. S. Sharp, O. Shakernia and S. S. Sastry, "A Vision System for Landing an Unmanned Aerial Vehicle," Proceedings of IEEE International Conference on Robotics and Automation (ICRA 2001), Seoul, Korea, May 2001, 
pp. 1720-1727.

[6] C. Akinli, "Semi-Autonomous Terminal Phase Spacecraft Docking Attitude Determination and Control," 2004. http://cengiz.akinli.org/research/vsgc-2004.pdf

[7] V. A. Grishin, "Precision Estimation of Camera Position Measurement Based on Docking Marker Observation," Pattern Recognition and Image Analysis, Vol. 20, No. 3, 2010, pp. 341-348.

[8] H. Cramer, "Mathematical Methods of Statistics," Princeton University Press, USA, 1946.
[9] I. A. Ibragimov and R. Z. Khasiminskii, "Asymptotic Estimation Theory," Nauka, Moscow, 1979.

[10] A. B. Kryanev and G. V. Lukin "Mathematical Methods for Processing Indeterminate Data," Fizmatlit, Moscow, 2003.

[11] H. L. Van Trees, "Detection, Estimation, and Modulation Theory, Part 1: Detection, Estimation, and Linear Modulation Theory," John Wiley \& Sons Inc., New York, 2001. 